\title{
Innovations
}

\section{Open Osmosis: \\ Library of Open Educational Resources (OER) for Medical Education}

\section{Authors}

Ersilia M. DeFilippis ${ }^{1,2, *}$; Thasin Jaigirdar ${ }^{2, *}$; Shiv M.

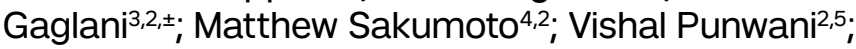
Rishi Desai ${ }^{2,6}$; M. Ryan Haynes ${ }^{2}$; Michael W. Painter ${ }^{7}$

\section{Abstract}

Many resources currently available for graduate and undergraduate medical education are fragmented, difficult to access, and costly. Osmosis, a web- and mobile-based platform, was developed to create a shared and easily accessible repository of curated clinical educational resources. We have recently launched "Open Osmosis" - a public-facing portal that has become one of the largest databases of Creative Commons-licensed questions, among other open educational resources (OER), for medical education. Initially created for use at the Johns Hopkins School of Medicine, Osmosis spread to recruit and involve medical students, physicians, and clinical content advisors from dozens of countries and hundreds of institutions. These individuals have served various roles, including as question-writers ("Medical Contributors"), content organizers ("Medical Scholars"), and expert reviewers ("Clinical Advisors"). Here we describe our experience developing Open Osmosis as a case study for crowdsourcing medical education content, and to comment on potential future development of this platform.

\section{Keywords}

medical education; open educational resources; crowdsourcing.

\section{Introduction}

As the breadth and depth of medical knowledge grows exponentially, clinical students often feel inundated with content they are expected to learn during their training. However, many of the available study resources are not clinically comprehensive, but instead are focused on a particular field, such as neurology or pathology, or the resources may be tailored to a specific exam, such as the United
'Brigham and Women's Hospital, Boston, MA, USA

${ }^{2}$ Osmosis - Knowledge Diffusion, Baltimore, MD, USA

${ }^{3}$ Johns Hopkins University School of Medicine, Baltimore, MD, USA

${ }^{4}$ Scripps Mercy Hospital, San Diego, CA, USA

${ }^{5}$ Khan Academy, Mountain View, CA, USA

${ }^{6}$ Stanford School of Medicine, Stanford, CA, USA

${ }^{7}$ Robert Wood Johnson Foundation, Princeton, NJ, USA

${ }^{*}$ Co-First Authors

^ Corresponding author: sgaglani@jhmi.edu
Cite this article as:

DeFilippis EM, Jaigirdar T, Gaglani SM, Sakumoto M, Punwani V, Desai R, Haynes MR, Painter MW. Open Osmosis: A Library of Open Educational Resources (OER) for Medical Education, Innovations in Global Health Professions Education. 2015:3 http://dx.doi.org/10.20421/ighpe2015.3

This is an open access article distributed under the terms of the Creative Commons Attribution license CC BY 4.0, which permits unrestricted use, distribution and reproduction in any medium, provided the original work is properly cited. 
States Medical Licensing Exam (USMLE). These resources vary in form, e.g. reference articles, practice questions, videos, images, and mnemonics. Subsequently, the information available to students is often fragmented, non-uniform, and difficult to access. This generally leads to low utilization rates and poor long-term knowledge retention among those who do use these resources.

One way to address this challenge is to develop a shared and easily accessible repository of curated clinical educational resources. Due to their ease of access and ubiquity, mobile apps and mobilecompatible websites are becoming increasingly prevalent in clinical student education ${ }^{2-6}$ and, thus, make a viable medium for this repository. Mobile educational tools range from reference solutions, such as Epocrates and Medscape, to social networking platforms, such as Figurel and Doximity. We recently described Osmosis (www.osmosis.org), a web- and mobile-learning platform that we (MRH, SMG) created to leverage crowdsourcing for improving access to medical education. ${ }^{7}$

Initially developed for use at the Johns Hopkins School of Medicine, Osmosis empowers students and teachers to create and curate educational content, such as questions, flashcards, videos, images, and mnemonics. To date, more than 1,000 students have created over 125,000 questions and flashcards within private Osmosis class groups that have been answered in excess of 5 million times. Overall, the platform is used by $30,000+$ clinical students around the globe, representing 500 institutions across 100 countries.

Given this scale of usage by clinical students, we realized there was a need to create a large database of open educational resources (OER) for medical education. Similar to Wikipedia, this content would be licensed under the Creative Commons Attribution Share-Alike (CC BY SA) license to promote the freely accessible "diffusion" of medical knowledge, ideally to even the most remote and under-resourced settings. With the generous support of the Robert Wood Johnson Foundation Pioneer Portfolio Program, we began developing this database of OER for medicine starting with the original development of thousands of openly accessible clinical questions that were structured as clinical vignettes, popularized by exams such as the USMLE.
In this paper we describe the process by which we developed this large database of OER, and discuss the future of the initiative, which we have named "Open Osmosis."

\section{Process}

We have previously described our original work in crowdsourcing medical education content, ${ }^{7}$ and have since developed a more robust understanding of the process and workflow needed to scale up production of OER for a global audience. Key priorities identified include: (1) setting content development priorities, (2) recruiting content contributors and editors, (3) training contributors to improve and standardize the quality of submissions, (4) reviewing and editing the submitted content, (5) publishing content, and (6) monitoring feedback to facilitate ongoing improvement.

In early 2014, it became clear that we would need more resources to complete these steps at scale, and thus we began searching for institutions that shared our vision and could provide resources to accomplish this goal. Based on its medical education-oriented grants to the Khan Academy and a consortium of medical schools led by Stanford, the Robert Wood Johnson Foundation (RWJF) was well-positioned to help kickstart the Open Osmosis initiative. In Spring 2014, we received a RWJF grant that allowed us to begin recruitment of contributors in May 2014. ${ }^{8}$

\section{Setting content development priorities}

Given our intended audience of clinical trainees primarily medical students around the world - we decided to focus on 12 key clinical areas based upon their common needs and feedback. These clinical areas were anesthesiology, dermatology, emergency medicine, neurology, obstetrics and gynecology, ophthalmology, osteopathic principles, pediatrics, psychiatry, radiology, spine and musculoskeletal medicine, and surgery. Our goal was to create approximately 5,000 clinical questions and to curate associated multimedia resources across these clinical disciplines.

\section{Recruitment of contributors and editors}

Four essential roles were created at the beginning of the initiative: Project Coordinator, Medical Scholar, 
Medical Contributor, and Clinical Advisor. The role of the Project Coordinator was to oversee the team and ensure that we were meeting appropriate milestones. All of the 12 Medical Scholars, all upper-level medical students or residents, were primarily responsible for one of the content areas. They ensured that the key topics and core concepts within a particular discipline were well represented and accurate. For example, the Medical Scholar in psychiatry was responsible for ensuring that there was an appropriate number of questions on child psychiatry, geriatric psychiatry, mood disorders, eating disorders, and other related areas. In addition, the Medical Scholar served as a leader of and advisor to the Medical Contributors in their respective disciplines, providing feedback and suggestions, as needed. Medical Contributors, generally upper level medical students (third- or fourth-year medical students in the United States medical education system), were primarily responsible for writing questions in a format similar to those found in medical licensing examinations, such as the USMLE. Clinical Advisors, primarily residents and medical school or hospital faculty members, were responsible for the final review of questions to ensure accuracy and relevance.

Once these roles were articulated, we launched a recruitment drive that directed potential contributors to an application website (https://www.osmosis.org/ oer/apply). The website explained the responsibilities and benefits of participation in the project. At the time, the Osmosis platform had $\sim 10,000$ medical student users, so initial recruitment efforts were focused on this cohort, as well as on their student and faculty networks. We also reached out to finalyear medical student classes, given their relatively flexible schedules and appropriate educational level for preparing this content. Finally, we contacted 25,000 United States medical school faculty to gauge their interest in participating in this opportunity, some of whom eventually became Clinical Advisors.

Three recruitment drives were launched, one in Summer 2014, one in Fall 2014, and the last one in Spring 2015. Once an application was submitted, we contacted the prospective contributor with our training material and expectations, and then encouraged him/her to submit two to three sample questions with multimedia. This served two purposes:
(1) assessing the quality of the contributions, and (2) determining the responsiveness of the applicant, and his or her ability to follow guidelines.

\section{Training to standardize and improve the quality of submissions}

Once an applicant was selected as a Medical Scholar or Medical Contributor, he or she was provided detailed training material, including a "High Quality Question Checklist" with examples of well-written questions, as well as video recordings in which we discussed our expectations and demonstrated how to submit questions via the Osmosis content development interface. This material was largely influenced by the National Board of Medical Examiner's (NBME) Item Writing Manual for Constructing Written Test Questions for the Basic \& Clinical Sciences. ${ }^{9}$ We also created a private Facebook group through which our contributors could post questions and share best practices.

In addition, some Medical Scholars held their own web conferences and wrote their own Osmosisaligned materials to manage their team of Medical Contributors.

\section{Reviewing and editing submissions}

Each submitted piece of content went through a three-step approval process. First, the item was submitted by a Medical Contributor who was thoroughly briefed on the High Quality Question Checklist. Second, the item was reviewed by the Medical Scholar who was primarily responsible for the pack of items. Finally, a Clinical Advisor would scan the question for any remaining issues. At each step of this process, the question could be flagged for issues, such as inconsistent formatting or incorrect information. After the content was vetted, it would be tagged by the Scholar or Project Coordinator as "Ready to Publish."

\section{Publishing and monitoring content feedback for continuous improvement}

The final steps in the content development process were publishing and monitoring. These iterative steps were meant to generate broad feedback beyond the core team by observing how the content was received by end-users. By the end of 2015, 
all of the content we developed will be published at the Open Osmosis website (open.osmosis.org). The Osmosis platform enables us to monitor both subjective feedback and objective interactions with the content. For example, learners can comment directly on the content through feedback forms, as well as rate the quality of each individual question on a four-point scale. This subjective, crowdsourced feedback can be used to identify potential errors so that the content can be improved. Objective interactions with the OER content can also be used to correct issues. One simple example is analyzing choice discrimination. Consider a multiple choice question with five answer options: if choices $A$ through $E$ are each selected $20 \%$ of the time, that is a poorly written question because there is no discrimination between the choices and, hence, the question is likely ambiguous or the choices are all correct. Conversely, if choice B is selected $100 \%$ of the time, that could mean that the other choices (distractors) are too implausible or not challenging enough, and, hence, the question is likely too easy. We are planning to apply techniques from item response theory (IRT) in the broader Osmosis platform to analyze user-behavior ${ }^{10}$ and identify under-performing content so that it can be reviewed and improved.

\section{Results}

\section{Overview}

During the 15-month period between July 2014 and October 2015, the Open Osmosis project recruited and trained a team of more than 150 medical students, residents, and faculty from 33 countries who wrote over 5,000 high-quality questions across 12 clinical disciplines (Table 1).

\section{Contributor team}

More than 450 individuals applied to contribute to the initiative. We selected 179 (39.8 percent) based on the strength of their applications, prior experience, ability to meet deadlines, and our own team's bandwidth. We had contributors across each medical school year, as well as some contributors who were in combined MD/ $\mathrm{PhD}$ programs and in residency programs (Table 2).

Contributors were able to contribute content to multiple disciplines, though we encouraged them to focus on one to two topic areas. Ultimately, each clinical discipline was assigned a team of Medical Contributors (team size ranged from 14 to 47) and, at least, one Medical Scholar and Clinical Advisor.

\begin{tabular}{|l|c|c|c|c|}
\hline Clinical Discipline & Contributors & Questions & Images & Videos \\
\hline Anesthesiology & 15 & 144 & 10 & 4 \\
\hline Dermatology & 17 & 506 & 942 & 25 \\
\hline Emergency Medicine & 47 & 653 & 376 & 154 \\
\hline Neurology & 45 & 660 & 295 & 151 \\
\hline OB/GYN & 19 & 497 & 46 & 6 \\
\hline Ophthalmology & 14 & 181 & 108 & 19 \\
\hline Osteopathic Principles \& Practice & 19 & 480 & 93 & 34 \\
\hline Pediatrics & 24 & 530 & 179 & 48 \\
\hline Psychiatry & 20 & 554 & 51 & 135 \\
\hline Radiology & 22 & 401 & 491 & 74 \\
\hline Spine \& Musculoskeletal & 20 & 115 & 46 & 49 \\
\hline Surgery & 43 & 412 & 320 & 148 \\
\hline Miscellaneous ** & 19 & 105 & 294 & 10 \\
\hline Total & $\mathbf{1 7 9 *}$ & $\mathbf{5 , 2 2 8}$ & $\mathbf{3 , 2 5 1}$ & $\mathbf{8 5 6}$ \\
\hline
\end{tabular}

Table 1. Summary statistics of the open educational resources produced per clinical discipline 
We observed that contributors who were most prolific in terms of the number of questions submitted were senior medical students (Table 3 ) with over 70 percent $(3,720$ of 5,228$)$ of our questions coming from this group. We believe that the senior medical student group may be ideal for crowdsourcing clinical content because (a) they are in their clinical years and, thus, typically have a greater knowledgebase than their junior peers, and (b) they may have more time to dedicate toward extra-curricular activities, such as content contribution. Fourth-year medical students may be particularly well-suited to contribution because they are typically less timeconstrained after applying for residencies.

\section{Productivitiy of the contributors and pace of work}

We observed a wide discrepancy in the productivity of invividual contributors (Figure 1). In previous work" we had found what we describe as an "80/20 split," whereby $80 \%$ of the content contributions were submitted by just $20 \%$ of the contributors. The disparity was slightly higher in this initiative, with the top $20 \%$ of the contributors responsible

\begin{tabular}{|c|c|c|c|c|c|}
\hline $\begin{array}{c}\text { First } \\
\text { Year (M1) }\end{array}$ & $\begin{array}{c}\text { Second } \\
\text { Year (M2) }\end{array}$ & $\begin{array}{c}\text { Third Year } \\
\text { (M3) }\end{array}$ & $\begin{array}{c}\text { Fourth } \\
\text { Year (M4) }\end{array}$ & MD/PhD & $\begin{array}{c}\text { Postgraduate } \\
\text { Years (PGY) }\end{array}$ \\
\hline 20 & 35 & 48 & 26 & 5 & 14 \\
\hline
\end{tabular}

Table 2. Year in training of $148^{*}$ selected contributors

*School-year data available for 148 of the 179 total medical student contributors

\begin{tabular}{|c|c|c|c|c|c|}
\hline $\begin{array}{c}\text { First } \\
\text { Year (M1) }\end{array}$ & $\begin{array}{c}\text { Second } \\
\text { Year (M2) }\end{array}$ & $\begin{array}{c}\text { Third Year } \\
\text { (M3) }\end{array}$ & $\begin{array}{c}\text { Fourth } \\
\text { Year (M4) }\end{array}$ & MD/PhD & $\begin{array}{c}\text { Postgraduate } \\
\text { Years (PGY) }\end{array}$ \\
\hline 488 & 736 & 1,626 & 2,094 & 110 & 102 \\
\hline
\end{tabular}

Table 3. Question submissions of $148^{*}$ selected contributors by year of medical school

*School-year data available for 148 of the 179 total medical student contributors

for $85 \%$ of the questions $(4,436)$, and the top $10 \%$ of contributors responsible for $74 \%(3,883)$. The bottom $50 \%$ of contributors created just $2.9 \%$ of the questions (151).

In addition to wide variation in productivity, there was nonlinear growth in the pace of contributions. The first recruitment drive brought on approximately 100 contributors who, over July and August 2014, contributed nearly 2,000 questions. Once the academic year resumed, however, there was a significant deceleration in the pace of work (Figure 2). We managed to accelerate the process at various points with additional recruitment drives and contribution competitions, but were most successful when we offered financial incentives to the top contributors.
Segment 1 corresponded to the taking on board of approximately 100 contributors and a bonus for contributing the most questions in July 2014. The acceleration in Segment 2 resulted from a bonus for writing more than $50+$ questions through mid-September. Segment 3 corresponded to the onboarding of more contributors, as well as a bonus for writing 20+ questions that month. And finally, segment 4 resulted primarily from incentivizing top contributors at a higher level than they were previously, once we had analyzed the data showing productivity discrepancies.

\section{Discussion}

The Open Osmosis initiative has successfully created and curated thousands of open educational 


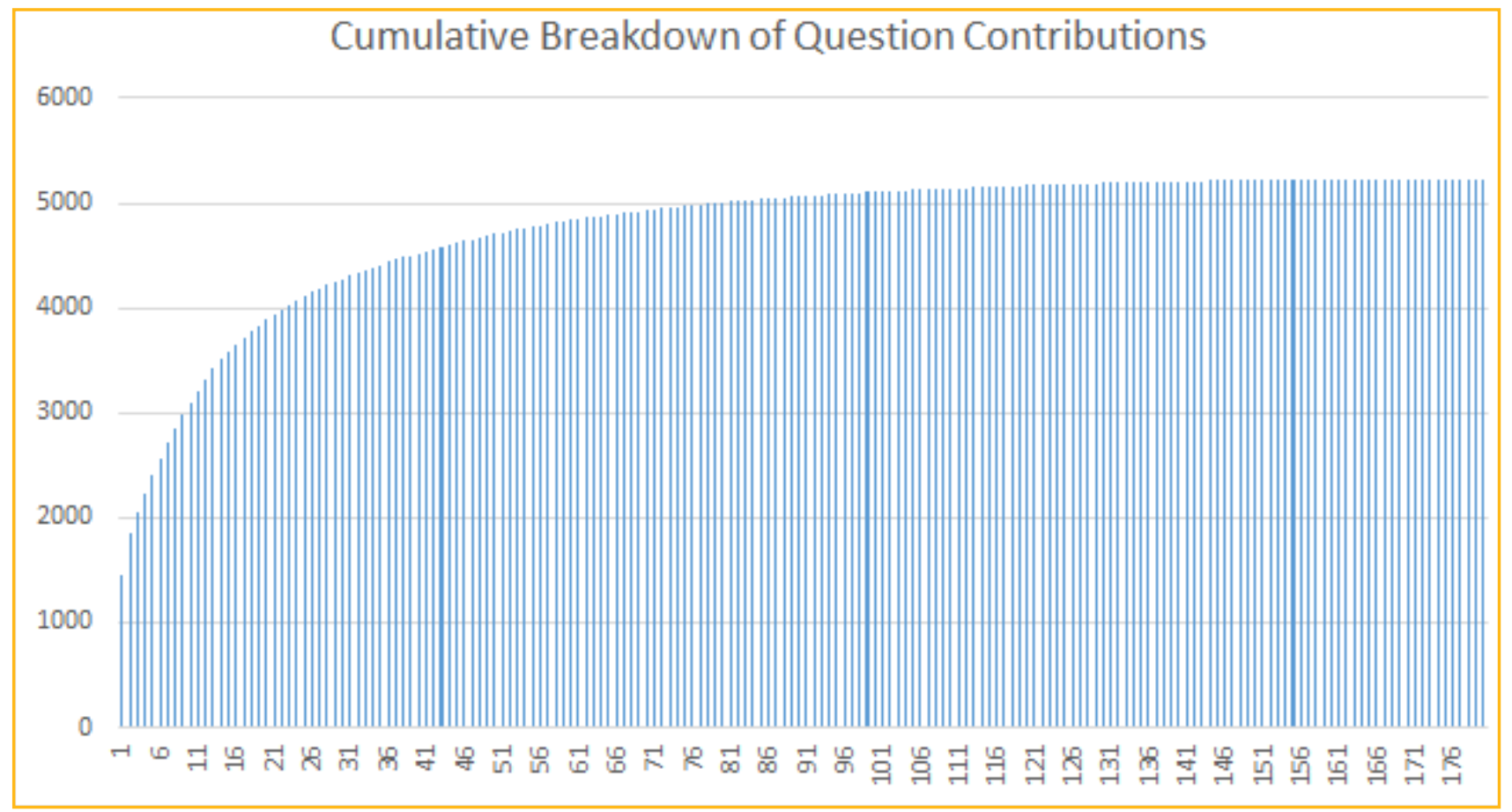

Figure 1. Cumulative number of questions contributed ( $y$-axis) by individual contributors ( $x$-axis). For example, contributor 1 submitted close to 1,500 questions while contributor 179 submitted 1 item.

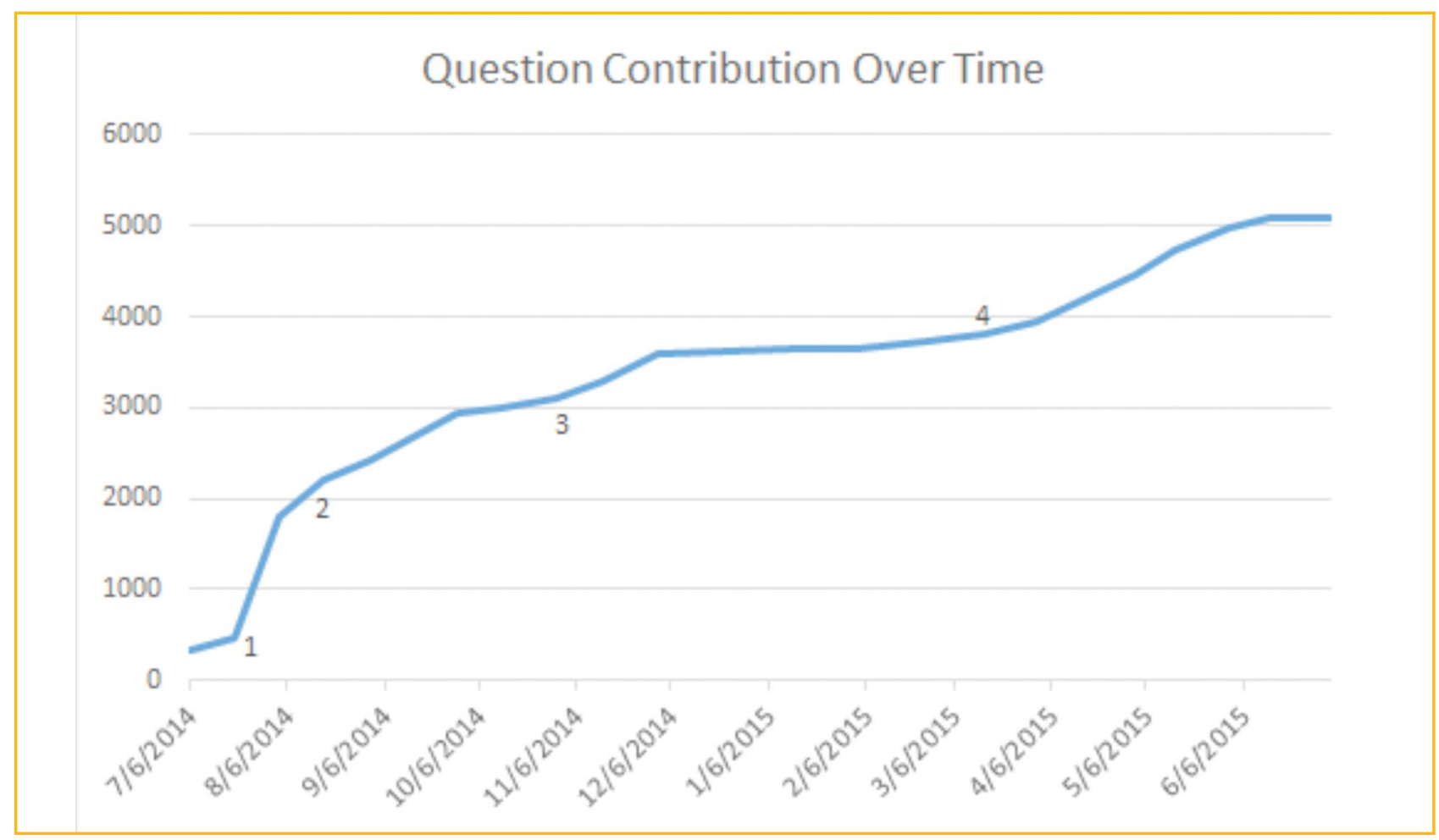

Figure 2. Pace of contributions to the OER question bank. 
resources (OER) for medical education. In addition to the inherent utility of these resources, the initiative empowered more than 150 clinical students, residents, and physicians to contribute to our shared mission of improving access to high-quality learning resources.

We found four common motivations that drove these individuals to contribute to the initiative. First and foremost, participation in the initiative enabled contributors to learn through peer-to-peer teaching. Online peer-to-peer teaching has a number of benefits beyond leveraging e-learning technologies, such as Osmosis. It has been shown to provide participants a more diverse medical education" and is thought to increase student confidence and competence. ${ }^{12}$ Furthermore, shared clinical learning experiences can be widely disseminated ${ }^{13}$ when clinical trainees from various countries are connected in a community. A student in one country may begin by writing a vignette that is subsequently reviewed by numerous other students across the world who can comment, make suggestions, and add relevant resources, including websites, videos, and mnemonics. For example, through the Open Osmosis initiative, a medical student in Mexico submitted a question that was reviewed by a clinical trainee in Ireland and then approved by a physician in the United States. Learning is shared among students with various educational experiences, enhancing the degree and accuracy of information available. Furthermore, participants benefit from the aspects of peer connectivity and social networking.

A second reason people contributed to the initiative was to help their peers and future learners. They were motivated by the mission to provide openly accessible educational resources, as Wikipedia and similar sites have done for millions of learners. A third motivation was to improve their resumes. Many of our contributors have listed participation in this initiative on their applications to residency, fellowship, and even clinical jobs. We have been able to quantify their "educational impact factor" by sharing data on their contributions that can make their impact on the initiative more tangible. The final common reason people participated was for the financial compensation, which for many helped defray costs incurred during their clinical training or applications to postgraduate training programs.

\section{Challenges encountered and mitigants}

We encountered both anticipated and unanticipated challenges throughout the process primarily related to two areas: quality and coordination. Logistically, given that the Open Osmosis initiative was a global project with Contributors, Scholars, and Advisors from 33 countries, it was difficult to coordinate conference calls to ensure that the team was working together seamlessly. As a consequence, we had variable commitment on the part of Scholars and Contributors. This led to large discrepancies in the quality of questions and resources submitted, which in turn required additional time to improve. In some cases lack of appropriate oversight by the Scholar led to gaps in content coverage. In particular, the Anesthesiology and Ophthalmology packs went through extended periods of time without oversight by a Scholar, and, thus, those packs had the fewest number of questions submitted. The academic calendars of our contributors also became an obstacle because clinical and curricular duties increased around the Fall months, which coincided with a reduction in contributions (Figure 2). Additionally, our use of financial incentives in some cases, paradoxically, led to reduced quality of contributions because these motivated lower-quality contributors to submit more content.

Though we distributed a High Quality Question Checklist as part of the training of new contributors, we found it necessary to update the checklist throughout the project. For example, based on learner feedback we decided one year into the project to mandate a "Major Takeaway" point for each question. The Major Takeaway is a 1-2 sentence summary of the question's major teaching point. As the utility of this summary became apparent at close to the midpoint of the project, we had to revise the thousands of questions submitted at that point to add this component to the question's main explanation. This would have been mitigated had we anticipated the need for this element in the checklist. Another challenge we found was that not all contributors adhered to the checklist as evidenced by improper formatting, as well as sourcing of images that were not licensed for open Creative Commons use. This latter issue led to approximately 1,000 images needing to be replaced at the end of the project due to inappropriate rights 
permissions. Because of the multi-tiered structure of the Osmosis team, Medical Scholars and Clinical Advisors were able to correct formatting issues and inaccuracies, but at a significant cost in terms of time and effort.

In addition to quality discrepancies between the contributors, we found significant productivity differences. The top ten percent of contributors contributed $74 \%$ of the questions and the bottom $50 \%$ of contributors contributed less than three percent of questions (Figure 1). The conclusion here is that, for future development efforts, instead of having a group of more than 150 contributors, it would be better to find the most dedicated group of contributors earlier and devote more time and resources to them. When we developed a program specifically targeting our top contributors in early 2015, we observed increased contributions by our writers (Figure 2), which remained consistent through the end of our question writing process.

One way to do this will be to screen potential contributors more stringently by not only continuing to include sample written questions with their applications, but also by evaluating the quality of their contributions earlier in the process. Many contributors had written a dozen or more questions before they were given feedback. Furthermore, many contributors may have ceased writing questions because of the lack of immediate feedback. Assigning more individuals to review questions in real-time would help ensure higher quality and more timely feedback. Another potential mitigant is to develop a mentoring system that pairs more experienced Medical Contributors or Medical Scholars with newer Medical Contributors to establish and reinforce expectations and facilitate troubleshooting earlier. More formal training in the form of videos or one-on-one video conferences may also help to mitigate the above issues.

\section{Future work}

While we have made much progress towards creating one of the largest databases of open educational resources for medical education, there remains much room for expanding the Open Osmosis initiative. The first step will be to begin our continuous improvement process based on learner feedback and behavior after publishing all of the questions by the end of 2015. This also will enable us to identify potential gaps in coverage to prioritize for future content development efforts.

We also have begun partnering with lower-resourced educational institutions to provide access to this web- and mobile-accessible repository of OER. For example, we have an existing collaboration with the Consortium of New Southern African Medical Schools (CONSAMs, www.osmosis.org/openeducation/consams) that provides students at their member institutions complimentary access to not only Open Osmosis material but also other aspects of the Osmosis platform.

Another future area of work is to broaden the reach of the OER material we host by developing content for students in other health professions, such as nursing, pharmacy, physical therapy, and dentistry. This will make the Open Osmosis platform even more useful for global health professions education. We are continuously recruiting contributors to the initiative and those interested can get in touch with us at hi@osmosis.org.

\section{Conclusion}

Through building upon our previous work in crowdsourcing, we have successfully launched the Open Osmosis initiative with thousands of open educational resources for medical education. This is an innovative and cost-effective educational program that demonstrates the benefits and potential impact of mobile- and web-based technologies in medical education. We intend to further grow the initiative and continue innovating to be able to provide open access to high quality educational material to future healthcare professionals around the world.

\section{Acknowledgements}

We would like to acknowledge the excellent contributions from the team of Medical Contributors, Medical Scholars, and Clinical Advisors who worked on this initiative. Finally, we would like to thank a number of clinical collaborators who contributed Creative Commons-licensed material through their successful websites: Dr. Amanda Oakley (DermNet $\mathrm{NZ}$ ), Dr. Brad Feldman and the American Academy of Ophthalmology (EyeWiki), Dr. Frank Gaillard (Radiopaedia), and Dr. Ross Donaldson (WikiEM). 


\section{Disclosure of interest}

MRH and SMG have formed a legal entity, Knowledge Diffusion, to promote the distribution of Osmosis.

\section{References}

1. Gaglani SM, Haynes MR. What can medical education learn from Facebook and Netflix? Ann Intern Med 2014;160(9):640-1.

2. Gaglani SM, Topol EJ. iMedEd: the role of mobile health technologies in medical education. Acad Med J Assoc Am Med Coll 2014;89(9):1207-9.

3. Lobo MJ, Crandley EF, Rumph JS, et al. Pilot Study of iPad Incorporation Into Graduate Medical Education. J Grad Med Educ 2013;5(1):142-4.

4. Niehaus W, Boimbo S, Akuthota V. Physical Medicine and Rehabilitation Resident Use of iPad Mini Mobile Devices. PM R 2015;

5. Nuss MA, Hill JR, Cervero RM, Gaines JK, Middendorf BF. Real-time use of the iPad by third-year medical students for clinical decision support and learning: a mixed methods study. J Community Hosp Intern Med Perspect 2014;4(4).

6. Boruff JT, Storie D. Mobile devices in medicine: a survey of how medical students, residents, and faculty use smartphones and other mobile devices to find information. J Med Libr Assoc JMLA 2014;102(1):22-30.

7. Haynes MR, Gaglani SM, Wilcox MV, Mitchell T, DeLeon V, Goldberg H. Learning through Osmosis: A collaborative platform for medical education. Innov Glob Med Health Educ 2014;2014(1):2.

8. Gaglani SM, Haynes MR, and Painter M. Another Step Toward Open Health Education. RWJF Culture of Health website. May 22, 2014. Available: http://www.rwjforg/en/culture-ofhealth/2014/05/another step toward.html.
9. Case SM and Swanson DB. Constructing Written Test Questions for the Basic and Clinical Sciences. Third Edition (revised). Accessed April 1, 2014. Available: http://www.nbme.org/publications/itemwriting-manual.html

10. Theobald J, Gaglani S, Haynes MR. The association between confidence and accuracy among users of a mobile web platform for medical education. Ann Intern Med 2015;162(5):395-6.

11. Keynejad R, Ali FR, Finlayson AET, et al. Telemedicine for peer-to-peer psychiatry learning between UK and Somaliland medical students. Acad Psychiatry J Am Assoc Dir Psychiatr Resid Train Assoc Acad Psychiatry 2013;37(3):182-6.

12. Information NC for B, Pike USNL of $M 8600$ R, MD B, Usa 20894. Peer-to-peer teaching: improving communication techniques for students in an accelerated nursing program. PubMed - NCBI [Internet]. [cited 2015 Mar 26]; Available: https://webvpn.med.cornell.edu.

13. Lynch NP, Cil T, Lehane E, Reardon M, Corrigan $M$. Transatlantic Peer-to-Peer Learning: An Initial Feasibility Analysis. Surg Innov 2014;21(6):643-8. 